\title{
Heart Sounds Obtained With Non-Contact Continuous-Wave Echo Doppler
}

\author{
Johannes J Struijk, Dagbjört H Eiriksdóttir, Ásgerdur A Pálsdóttir, Samuel E Schmidt \\ Aalborg University, Aalborg, Denmark
}

\begin{abstract}
Heart sounds can be obtained in various ways. A recent modality is airborne ultrasound echo Doppler, which has been used either in a pulsed wave mode or a continuous wave mode. In the present work we analyse the continuous wave ultrasound echo from the chest.

In 10 subjects a piezo ultrasound transceiver was used to transmit a $40 \mathrm{kHz}$ acoustic sine wave directed to the chest wall. The reflected signal (echo) was recorded with a microphone and sampled at $1 \mathrm{MHz}$. The FM modulated echo was demodulated in MatLab using a quadrature signal.

The signal thus obtained represented the chest displacement, which was subdivided into three frequency bands: respiration (below $0.5 \mathrm{~Hz}$ ), movement of the myocardium (>1 Hz) and valve sounds $(30-100 \mathrm{~Hz})$. Above $100 \mathrm{~Hz}$ the signal decayed by $20 \mathrm{~dB} /$ decade with a noise floor above 1-2 kHz. Interestingly, signals obtained directly from the skin and signals obtained through a thin layer of clothes were similar.

Non-contact continuous-wave echo Doppler thus is a promising new modality for the recording of heart sounds, although its true potential has to be further investigated.
\end{abstract}

\section{Introduction}

Acoustic echo-Doppler is a well-known method for the measurement of velocity of a moving object and can be used to measure vibrations with very small amplitudes as well [1]. Recently, some work appeared in the literature on the recording of chest movement induced by the beating heart using continuous wave Doppler (CWD) [2] and by pulsed wave Doppler (PWD) [3,4] as well.

Although CWD easily runs into problems of continuous (!) echos from all sorts of reflectors in the environment, one of its main advantages, as compared with PWD, is that very high sample rates can be obtained, whereas PWD is limited by the time-of-flight of the signal.

We therefore used CWD as our method to record vibrations from the chest and we analysed the resulting signals in terms of respiration, myocardial movement and valve sounds.

\section{Methods}

A 400EP250 Prowave ultrasound transmitter was used to generate a continuous $40 \mathrm{kHz}$ acoustic sine wave directed to the chest wall. The waveform generator of a Keysight EDUX1002G oscillisocope was directly connected to the transmitter for this purpose. The reflected signal (echo) was recorded using a CM16/CMPA ultrasound microphone, a $116 \mathrm{H}$ US gate and USGH recording software from Avisoft using a sampling frequency of $1 \mathrm{MS} / \mathrm{s}$.

If the emitted signal is given as

$$
\text { (1) } s_{e}(t) \sim \cos \left(2 \pi f_{c} t\right) \text {, }
$$

then the reflected signal can be written as

$$
s_{e}(t) \sim \cos \left(2 \pi f_{c}\left(t+\frac{2}{c} d(t)\right)\right)
$$

where $f_{c}$ is the carrier frequency ( $40 \mathrm{kHz}$ ), $c$ is the velocity of sound in air $(\approx 345 \mathrm{~m} / \mathrm{s})$ and $d(t)$ is the displacement of the skin of the chest (see fig. 1).

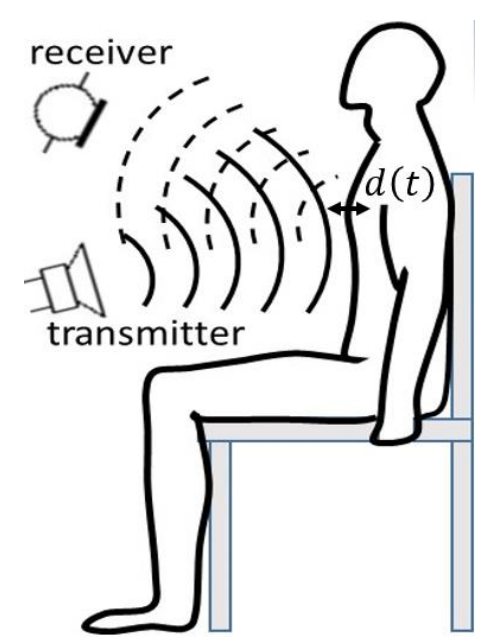

Figure 1. The essential parts of the measurement setup. 
According to equation (2) the echo signal consists of the $40 \mathrm{kHz}$ carrier which is phase/frequency modulated by the displacement of the chest wall. FM demodulation, directly to baseband, was obtained in MatLab using a standard quadrature method and the signal was then resampled at 5 $\mathrm{kHz}$.

Differentiation of the resulting displacement signal gave the velocity and a second differentiation yielded the acceleration. The latter was compared with a seismocardiographic signal obtained using an accelerometer that was positioned on the xiphoid process of the sternum, whereas the acoustic wave was directed at an area somewhat above the accelerometer. The acoustic signal was analysed in three frequency bands to obtain respiration, myocardial motion and valve sounds simultaneously.

\section{Results}

Figure 2 shows the unfiltered displacement signal of a duration of $46 \mathrm{~s}$, clearly showing six respiratory cycles with amplitudes of approximately $6 \mathrm{~mm}$ peak to peak. The signal was obtained with the piezo transmitter and the microphone at $10 \mathrm{~cm}$ distance from the chest.

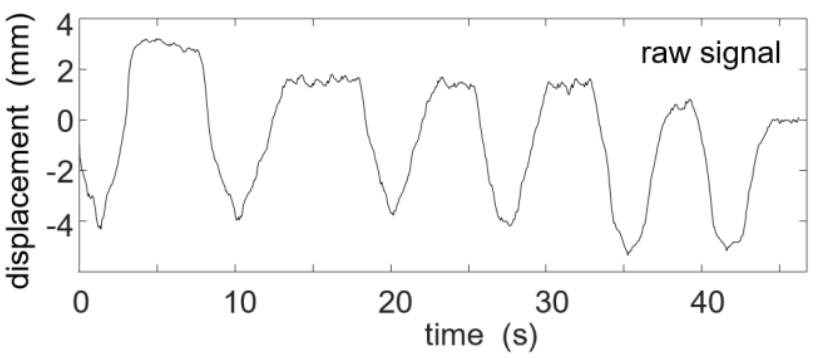

Figure 2. The unfiltered displacement signal.

Removing the respiratory component of the signal using a high-pass filter with a cut-off frequency of $0.5 \mathrm{~Hz}$ resulted in the signal shown in figure 3, upper graph. This signal had a varying amplitude, mainly depending on the respiration and typically in the order of tenths of millimeters.

Clear valve sounds are seen in the $30-100 \mathrm{~Hz}$ band (fig. 3 middle graph) and typically have an amplitude of a few micrometers. A wider bandwidth of $15-100 \mathrm{~Hz}$ may increase the amplitude, but it is yet unclear how much the movement of the myocardium then contaminates the valve sounds. It is noticable that the noise level is below $0.1 \mu \mathrm{m}$, which is in accordance with the analysis given by Cretin et al. [1].

For reasons of comparison the acceleration signal, derived from the displacement, is shown in fig. 3 lower graph, lower trace. Double differentiation introduces a high noise level as compared to a direct measurement of the acceleration with an accelerometer. Nevertheless, the similarity between the signals is striking. The difference in amplitudes might be caused by the different measurement points/areas on the chest, where the accelerometer is positioned relatively low relative to the heart.

With the measurement apparatus at greater distances from the chest and when the volunteers wore a T-shirt, the signals tended to lose some quality in terms of noise and artifacts, although in many cases the signal was very similar to the measurements at $10 \mathrm{~cm}$ and without shirt.
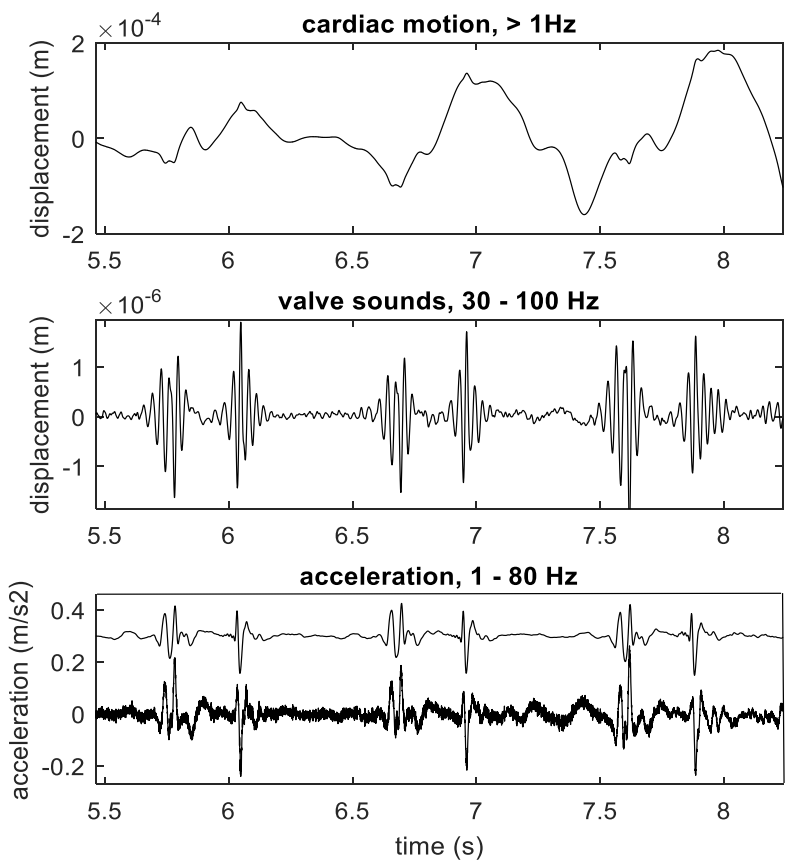

Figure 3. Upper and middle graph: displacement signals in two frequency bands containing the myocardial movement and the valve sounds respectively. Lower graph: acceleration as derived from the CWD echo (lower trace) and from the accelerometer (upper trace - with offset to visually separate the signals).

An analysis of the frequency spectrum of the CWD signal shows a $20 \mathrm{~dB} /$ decade characteristic and a noise floor above 1 or $2 \mathrm{kHz}$, thus promising a potential for murmur detection (up to $800 \mathrm{~Hz}$ ) as well.

\section{Conclusion}

The ability of the CWD method to measure very small displacements (submicron) in a very wide frequency band from less than $0.1 \mathrm{~Hz}$ to beyond $1 \mathrm{kHz}$ makes it a valuable tool to simultaneously record all the relevant respiratory and cardiac acoustic signals.

\section{References}

[1] Cretin B, Vairac P, Jachez N, Pergaud J. Sensitive ultrasonic vibrometer for very low frequency applications. Rev Sci Instr 2007;78:085112. 
[2] Kranjec J, Begus S, Drnovsek J, Gersak G. Novel methods for noncontact heart rate measurement: A feasibility study. IEEE Trans Instr Meas 2014;63:838-847.

[3] Jeger-Madiot N, Gateau J, Fink M, Ing RK, Non-contact and through-clothing measurement of the heart rate using ultrasound vibrocardiography. Med Eng Phys 2017;50:96102.

[4] Shirkovskiy P, Laurin A, Chapelle D, Fink M, Ing RK. Contactless mapping of thoracic and abdominal motion: applications for seismocardiography. Comput Cardiol 2017.
Address for correspondence.

Johannes J. Struijk.

Cardiotechnology Research Group

Department of Health Science and Technology

Aalborg University

Fredrik Bajersvej 7C1-205

9220 Aalborg

Denmark

jjs@hst.aau.dk. 